\title{
On local times of a symmetric stable process as a doubly indexed process
}

\author{
NATHALIE EISENBAUM \\ Laboratoire de Probabilités, Université Paris VI, 4 Place Jussieu, Case 188, 75252 Paris Cedex \\ 05, France.E-mail: nae@ccr.jussieu.fr
}

We consider the local time process $\left(L_{t}^{x}, x \in \mathbb{R}, t \geqslant 0\right.$ ) of a symmetric stable process $X$ with index $\beta$ in $(1,2]$. We compute the $p$-variation of $L$ on any rectangle of $\mathbb{R} \times[0, \infty)$. Unlike for the $p$-variation of $L$ with respect to the spatial parameter (studied by Marcus and Rosen), we show here that the Brownian case - when $\beta=2$ - is atypical.

Keywords: Itô formula; local time; $p$-variation; symmetric stable process

\section{Introduction and notation}

Let $\left(X_{t}, t \geqslant 0\right)$ be a symmetric stable process with index $\beta$ in $(1,2]$. This means that $X$ is a real-valued process with stationary independent increments such that

$$
\mathrm{E}\left[\mathrm{e}^{i \alpha X_{t}}\right]=\mathrm{e}^{-t|\alpha|^{\beta}}, \quad \alpha \in \mathbb{R} .
$$

This process admits a continuous local time process $\left(L_{t}^{x}, x \in \mathbb{R}, t \geqslant 0\right.$ ) (see Boylan 1964; Barlow 1988).

Marcus and Rosen (1992b) have shown that if $\left(\pi_{n}\right)_{n \in \mathbb{N}}$ is any sequence of partitions of $[a, b]$, a subinterval of $\mathbb{R}$, such that $\left|\pi_{n}\right|$ converges to 0 as $n$ tends to $\infty$, then uniformly in $t$ on any bounded subinterval of $\mathbb{R}^{+}$, for all $r>0$,

$$
\sum_{x_{i} \in \pi_{n}}\left|L_{t}^{x_{i+1}}-L_{t}^{x_{i}}\right|^{2 /(\beta-1)} \underset{n \rightarrow \infty}{\stackrel{L^{r}}{\longrightarrow}} c(\beta) \int_{a}^{b}\left|L_{t}^{x}\right|^{1 /(\beta-1)} \mathrm{d} x,
$$

where $c(\beta)$ is a constant that only depends on $\beta$. For $\beta=2$, which is the case when $X$ is a Brownian motion, (1) was already established by Bouleau and Yor (1981).

In this paper, we consider the local times as a doubly indexed process. For $[a, b]$ a subinterval of $\mathbb{R}$ and $[s, t]$ a subinterval of $[0,+\infty)$, let $\left(\Delta_{k}\right)_{k \in \mathbb{N}}$ be a sequence of grids of $[a, b] \times[s, t]$. For each $k$, write $\Delta_{k}=\left\{\left(x_{i}, t_{j}\right), 1 \leqslant i \leqslant n, 1 \leqslant j \leqslant m\right\}$, where, for notational simplicity, we drop the dependence on $k$. Suppose that $\left|\Delta_{k}\right|$ converges to 0 as $k$ tends to $\infty$. We seek the limit in $L^{1}$ as $k$ tends to $\infty$ of the sum

$$
\sum_{\left(x_{i}, t_{j}\right) \in \Delta_{k}}\left|L_{t_{j+1}}^{x_{i+1}}-L_{t_{j}}^{x_{i+1}}-L_{t_{j+1}}^{x_{i}}+L_{t_{j}}^{x_{i}}\right|^{2 /(\beta-1)} .
$$

The process $L$ has been studied as a doubly indexed process by Walsh (1983), Rogers and 
Walsh (1991) and Eisenbaum (1998). These authors have developed the notion of stochastic integration with respect to $L$ of doubly indexed processes. We will use some results of Eisenbaum (1998) in Section 4.

In Section 2, we treat the case $\beta \in(1,2)$. Sections 3 and 4 are devoted to the case $\beta=2$. This is an atypical case, because it is the only value of $\beta$ for which the limit in (2), for $s=0$, is equal to the limit in (1): when $X$ is a Brownian motion, we have

$$
\sum_{\left(x_{i}, t_{j}\right) \in \Delta_{k}}\left|L_{t_{j}+1}^{x_{i}+1}-L_{t_{j}}^{x_{i+1}}-L_{t_{j+1}}^{x_{i}}+L_{t_{j}}^{x_{i}}\right|^{2} \underset{k \rightarrow \infty}{\stackrel{L^{1}}{\longrightarrow}} 4 \int_{a}^{b}\left(L_{t}^{x}-L_{s}^{x}\right) \mathrm{d} x .
$$

Actually $\beta=2$ is the only case for which the local time process satisfies Tanaka's formula. The computations can hence be done by considering the process

$$
M(x, t)=\int_{0}^{t} 1_{\left(X_{s} \leqslant x\right)} \mathrm{d} X_{s} .
$$

In Section 3, we compute the quadratic variation of $L$. In Section 4, we exploit the process $M$ in order to rewrite, from a new point of view, an Itô formula obtained in Eisenbaum (1998).

For ease of notation, we will write, for any doubly indexed process $(Y(x, t), x \in \mathbb{R}$, $t \geqslant 0)$,

$$
\Delta_{i j} Y=\left(Y_{\left(x_{i+1}, t_{j+1}\right)}-Y_{\left(x_{i+1}, t_{j}\right)}-Y_{\left(x_{i}, t_{j+1}\right)}+Y_{\left(x_{i}, t_{j}\right)}\right) .
$$

Further, we let $\left(p_{t}(y), t \geqslant 0, y \in \mathbb{R}\right)$ be the transition densities of $X$ with respect to the Lebesgue measure and $\left(\mathscr{F}_{t}\right)_{t \geqslant 0}$ be the natural filtration of $X$. Finally, $c(\beta)$ will denote a generic constant depending only on $\beta$, which may be different from line to line.

\section{2. $p$-variation of the local times for $\beta$ in $(1,2)$}

We have the following theorem.

Theorem 2.1. For $\beta \in(1,2)$, let $\left(\Delta_{k}\right)_{k \in N}$ be a sequence of grids of $[a, b] \times[s, t]$, where, for each $k, \Delta_{k}=\left\{\left(x_{i}, s_{j}\right), 1 \leqslant i \leqslant n, 1 \leqslant j \leqslant m\right\}$. We suppose that $\left|\Delta_{k}\right|$ and $\sup _{\left(x_{i}, s_{j}\right) \in \Delta k} \mid x_{i+1}-$ $x_{i} \mid /\left(t_{j+1}-t_{j}\right)^{1 / \beta}$ both converge to 0 as $k$ tends to $\infty$. We then have

(i) $\sum_{(i, j)}\left|\Delta_{i j} L\right|^{2 /(\beta-1)} \underset{k \rightarrow \infty}{\stackrel{L^{1}}{\longrightarrow}} 0$,

(ii) $\mathrm{E}\left(\sum_{(i, j)}\left|\Delta_{i j} L\right|^{\beta /(\beta-1)}\right) \underset{k \rightarrow \infty}{\longrightarrow}+\infty$.

In view of Theorem 2.1, it is natural to ask whether there exists a critical exponent $\alpha$ such that we would have convergence of $\sum_{(i, j)}\left|\Delta_{i j} L\right|^{\alpha}$ in $L^{1}$ to a finite, non-zero limit. The answer is negative and given by the following proposition. We write $\mathscr{D}$ for the set of the grids of $[a, b] \times[s, t]$ satisfying the hypotheses of Theorem 2.1 . 
Proposition 2.2. For any $p \in(\beta /(\beta-1), 2 /(\beta-1))$, we have
(i) $\inf \left\{\mathrm{E}\left(\sum_{\left(x_{i}, t_{j}\right) \in \Delta}\left|\Delta_{i j} L\right|^{p}\right) ; \Delta \in \mathscr{D}\right\}=0$,
(ii) $\sup \left\{\mathrm{E}\left(\sum_{\left(x_{i}, t_{j}\right) \in \Delta}\left|\Delta_{i j} L\right|^{p}\right) ; \Delta \in \mathscr{D}\right\}=+\infty$.

Proof of Theorem 2.1. Define, for a fixed $y \in \mathbb{R}$ and a fixed $j$,

$$
d(i, j, y)=\frac{x_{i}-y}{\left(t_{j+1}-t_{j}\right)^{1 / \beta}} .
$$

(i) By successively applying the Markov property of $X$ at time $t_{j}$ and its scaling property, we obtain

$$
\begin{aligned}
\mathrm{E}\left[\sum_{(i, j)}\left|\Delta_{i j} L\right|^{2 /(\beta-1)}\right] & =\mathrm{E}\left[\sum_{(i, j)}\left|L_{t_{j+1}-t_{j}}^{x_{i+1}}-L_{t_{j+1}-t_{j}}^{x_{i}}\right|^{2 /(\beta-1)} \circ \theta_{t_{j}}\right] \\
& =\sum_{j} \mathrm{E}\left[\mathrm{E}\left[\left(\sum_{i}\left|L_{t_{j+1}-t_{j}}^{x_{i+1}}-L_{t_{j+1}-t_{j}}^{x_{i}}\right|^{2 /(\beta-1)}\right) \circ \theta_{t_{j}} \mid \mathscr{F} t_{j}\right]\right] \\
& =\sum_{j} \int_{\mathbb{R}} P\left(X_{t_{j}} \in \mathrm{d} y\right) \mathrm{E}\left[\sum_{i}\left|L_{t_{j+1}-t_{j}}^{x_{i+1}-y}-L_{t_{j+1}-t_{j}}^{x_{i}-y}\right|^{2 /(\beta-1)}\right] \\
& =\sum_{j}\left(t_{j+1}-t_{j}\right)^{2 / \beta} \int_{\mathbb{R}} P\left(X_{t_{j}} \in \mathrm{d} y\right) \sum_{i} \mathrm{E}\left[\left|L_{1}^{d(i+1, j, y)}-L_{1}^{d(i, j, y)}\right|^{2 /(\beta-1)}\right] .
\end{aligned}
$$

Consequently, we have

$$
\begin{aligned}
& \mathrm{E}\left[\sum_{(i, j)}\left|\Delta_{i j} L\right|^{2 /(\beta-1)}\right] \\
& \quad \leqslant \sup _{j}\left|t_{j+1}-t_{j}\right|^{2 / \beta-1} \sum_{j}\left(t_{j+1}-t_{j}\right) \int_{\mathbb{R}} P\left(X_{t_{j}} \in \mathrm{d} y\right) \sum_{i} \mathrm{E}\left[\left|L_{1}^{d(i+1, j, y)}-L_{1}^{d(i, j, y)}\right|^{2 /(\beta-1)}\right] .
\end{aligned}
$$

We note that since $2 / \beta>1$, we have

$$
\sup _{j}\left|t_{j+1}-t_{j}\right|^{2 /(\beta-1)} \underset{k \rightarrow \infty}{\longrightarrow} 0 .
$$

Hence, to prove (i) it is sufficient to show that

$$
\sum_{j}\left(t_{j+1}-t_{j}\right) \int_{\mathbb{R}} P\left(X_{t_{j}} \in \mathrm{d} y\right) \sum_{i} \mathrm{E}\left[\left|L_{1}^{d(i+1, j, y)}-L_{1}^{d(i, j, y)}\right| 2 /(\beta-1)\right]
$$

is uniformly bounded. 
We will use the following inequality, established by Marcus and Rosen (1992b): for any $p \geqslant 2$,

$$
\mathrm{E}\left[\left|\mathrm{L}_{t}^{x}-L_{t}^{y}\right|^{p}\right] \leqslant C(\beta, p) t^{(\beta-1) p / 2 \beta}|x-y|^{(\beta-1) p / 2},
$$

where $C(\beta, p)$ is a constant depending only on $\beta$ and $p$. Note that a direct use of the above inequality does not provide the uniform majorization that we want to establish.

Now note that, uniformly in $y$ and $j$,

$$
\sup _{i}(d(i+1, j, y)-d(i, j, y)) \underset{k \rightarrow \infty}{\longrightarrow} 0 .
$$

We set

$$
S_{1}=\sup _{0 \leqslant t \leqslant 1} X_{t}
$$

Let $(p, q)$ be a pair of positive real numbers such that $1 / p+1 / q=1$ and $1<q<\beta$. By successively applying Hölder's inequality and the above result of Marcus and Rosen, we obtain

$$
\begin{aligned}
& \mathrm{E}\left[\sum_{d(i, j, y) \geqslant 0}\left|L_{1}^{d(i+1, j, y)}-L_{1}^{d(i, j, y)}\right|^{2 /(\beta-1)}\right] \\
& \quad=\mathrm{E}\left[\sum_{d(i, j, y) \geqslant 0}\left|L_{1}^{d(i+1, j, y)}-L_{1}^{d(i, j, y)}\right|^{2 /(\beta-1)} ; S_{1} \geqslant d_{i}\right] \\
& \quad \leqslant \sum_{d(i, j, y) \geqslant 0} \mathrm{E}\left[\left(L_{1}^{d(i+1, j, y)}-L_{1}^{d(i, j, y)}\right)^{2 /(\beta-1)}\right]{ }^{1 / p} P\left[S_{1} \geqslant d(i, j, y)\right]^{1 / q} \\
& \quad \leqslant c(\beta) \sum_{d(i, j, y) \geqslant 0}(d(i+1, j, y)-d(i, j, y)) P\left[S_{1} \geqslant d(i, j, y)\right]^{1 / q} \\
& \quad=c(\beta) \sum_{n \in \mathbb{N}} \sum_{n \leqslant d(i, j, y)<n+1}(d(i+1, j, y)-d(i, j, y)) P\left[S_{1} \geqslant d(i, j, y)\right]^{1 / q} \\
& \quad \leqslant c(\beta) \sum_{n \in \mathbb{N}} P\left[S_{1} \geqslant n\right]^{1 / q} .
\end{aligned}
$$

Next we use the estimate (see, for example, Bertoin 1996, p. 221)

$$
P\left[S_{1} \geqslant x\right] \sim \frac{c(\beta)}{x^{\beta}}, \quad \text { as } x \rightarrow \infty .
$$

Since $\beta / q$ is strictly greater than 1 , we obtain by symmetry that

$$
\mathrm{E}\left[\sum_{i}\left(L_{1}^{d(i+1, j, y)}-L_{1}^{d(i, j, y)}\right)^{2 /(\beta-1)}\right] \leqslant 2 c(\beta) \sum_{n \in \mathbb{N}} P\left[S_{1} \geqslant n\right]^{1 / q}<\infty .
$$


Consequently,

$$
\sum_{j}\left(t_{j+1}-t_{j}\right) \int_{\mathbb{R}} P\left(X_{t_{j}} \in \mathrm{d} y\right) \sum_{i} \mathrm{E}\left[\left|L_{1}^{d(i+1, j, y)}-L_{1}^{d(i, j, y)}\right| 2 /(\beta-1)\right] \leqslant c(\beta)(t-s)<\infty .
$$

(ii) Similarly, we have

$$
\begin{aligned}
\mathrm{E}\left[\sum_{(i, j)}\left|\Delta_{i j} L\right|^{\beta /(\beta-1)}\right] & =\sum_{j}\left(t_{j+1}-t_{j}\right) \int_{\mathbb{R}} P\left(X_{t_{j}} \in \mathrm{d} y\right) \mathrm{E}\left[\sum_{i}\left|L_{1}^{d(i+1, j, y)}-L_{1}^{d(i, j, y)}\right| \beta /(\beta-1)\right] \\
& =\int_{\mathbb{R}} \int_{s}^{t} G_{k}(u, y) \mathrm{d} u \mathrm{~d} y,
\end{aligned}
$$

with

$$
G_{k}(u, y)=\sum_{j} 1_{\left(t_{j}, t_{j+1}\right]}(u) p_{t_{j}}(y) \mathrm{E}\left[\sum_{i}\left|L_{1}^{d(i+1, j, y)}-L_{1}^{d(i, j, y)}\right| \beta /(\beta-1)\right] .
$$

For a fixed pair $(u, y)$, there exists a unique sequence $(j(k))_{k}$ (actually $\left.(j(k, u))_{k}\right)$ such that $t_{j(k)}<u \leqslant t_{j(k)+1}$ for every $k$. Now if $y \in[a, b]$, then $(d(i, j(k), y))_{1 \leqslant i \leqslant n}$ is a partition such that $\sup _{i}(d(i+1, j(k), y)-d(i, j(k), y))$ tends to $0, d(1, j(k), y) \rightarrow-\infty$, and $d(n, j(k), y) \rightarrow+\infty$, as $k$ tends to $\infty$. Thanks to (1), we immediately have, for any $r>0$,

$$
\lim _{k \rightarrow \infty} \mathrm{E}\left[\sum_{|d(i, j(k), y)| \leqslant r}\left|L_{1}^{d(i+1, j(k), y)}-L_{1}^{d(i, j(k), y)}\right| \beta /(\beta-1)\right]=+\infty .
$$

Hence $G_{k}(u, y)$ converges to $\infty$ on $[s, t] \times[a, b]$ almost everywhere with respect to Lebesgue measure. By the Lebesgue dominated convergence theorem, this implies that, for any $M>0$,

$$
\int_{a}^{b} \int_{s}^{t} 1_{\left(G_{k}>M\right)} \mathrm{d} y \mathrm{~d} u \underset{k \rightarrow \infty}{\longrightarrow}(b-a)(t-s)
$$

Since

$$
\int_{\mathbb{R}} \int_{s}^{t} G_{k}(u, y) \mathrm{d} u \mathrm{~d} y \geqslant M \int_{a}^{b} \int_{s}^{t} 1_{\left(G_{k}>M\right)} \mathrm{d} y \mathrm{~d} u,
$$

we obtain that, for any $M>0$,

$$
\liminf _{k \rightarrow \infty} \int_{\mathbb{R}} \int_{s}^{t} G_{k}(u, y) \mathrm{d} u \mathrm{~d} y \geqslant M(b-a)(t-s) .
$$

Consequently,

$$
\lim _{k \rightarrow \infty} \int_{\mathbb{R}} \int_{s}^{t} G_{k}(u, y) \mathrm{d} u \mathrm{~d} y=+\infty .
$$


Proof of Proposition 2.2. (i) Let $\left(\epsilon_{k}\right)_{k \in \mathbb{N}}$ be a sequence of real numbers strictly decreasing to 0 . For each $k$, we set $n=\left[\left(b-a / \epsilon_{k}\right)\right]$ and we define the grid $\Delta_{k}$ by

$$
x_{1}=a, \quad x_{i+1}-x_{i}=\epsilon_{k}, 1 \leqslant i \leqslant n-2, \quad x_{n}=b .
$$

Now let $q$ be such that $0<q<\beta$. We set $m=\left[(b-a) / \epsilon_{k}^{q}\right]$. Similarly, we define

$$
t_{1}=s, \quad t_{j+1}-t_{j}=\epsilon_{k}^{q}, 1 \leqslant j \leqslant m-2, \quad t_{m}=t .
$$

With this definition of $\Delta_{k}$, the assumptions of Theorem 2.1 are readily verified and, moreover, we have

$$
\sup _{j}\left(t_{j+1}-t_{j}\right) \leqslant 3 \epsilon_{k}^{q} \text { and } \sup _{i, j} \frac{x_{i+1}-x_{i}}{\left(t_{j+1}-t_{j}\right)^{1 / \beta}} \leqslant 3 \epsilon_{k}^{1-q / \beta} .
$$

Following the same steps as in the proof of Theorem 2.1(i), we obtain

$$
\begin{aligned}
\mathrm{E} \sum_{(i, j)}\left|\Delta_{i j} L\right|^{p} & =\sum_{j}\left(t_{j+1}-t_{j}\right)^{p(\beta-1) / \beta} \int_{\mathbb{R}} P\left(X_{t_{j}}<\mathrm{d} y\right) \sum_{i} \mathrm{E}\left[\left|L_{1}^{d(i+1, j, y)}-L_{1}^{d(i, j, y)}\right| p\right] \\
& \leqslant \sup _{j}\left(t_{j+1}-t_{j}\right)^{p(\beta-1) / \beta-1} \sum_{j}\left(t_{j+1}-t_{j}\right) \int_{\mathbb{R}} P\left(X_{t_{j}} \in \mathrm{d} y\right) \sum_{i} \mathrm{E}\left[\left|L_{1}^{d(i+1, j, y)}-L_{1}^{d(i, j, y)}\right| p\right] .
\end{aligned}
$$

We retain the notation $S_{1}=\sup _{0 \leqslant s \leqslant 1} X_{s}$. For $\mu$ and $v$ positive real numbers such that $1 / \mu+1 / \nu=1$ and $1<\nu<\beta$, we have

$$
\begin{aligned}
& \sum_{d(i, j, y) \geq 0} \mathrm{E}\left[\left|L_{1}^{d(i+1, j, y)}-L_{1}^{d(i, j, y)}\right| p\right] \\
& \leqslant \sum_{d(i, j, y) \geqslant 0} \mathrm{E}\left[\left|L_{1}^{d(i+1, j, y)}-L_{1}^{d(i, j, y)}\right| p \mu\right] 1 / \mu P\left[S_{1} \geqslant d(i, j, y)\right]^{1 / v} \\
& \leqslant c(\beta) \sum_{d(i, j, y) \geqslant 0}(d(i+1, j, y)-d(i, j, y))^{(\beta-1) p / 2} P\left[S_{1} \geqslant d(i, j, y)\right]^{1 / v} \\
& \leqslant c(\beta) \sup _{d(i, j, y) \geqslant 0}(d(i+1, j, y)-d(i, j, y))^{(\beta-1) p / 2-1} \sum_{d(i, j, y) \geqslant 0}(d(i+1, j, y)-d(i, j, y)) \\
& \times P\left[S_{1} \geqslant d(i, j, y)\right]^{1 / v} .
\end{aligned}
$$

As we have already seen in the proof of Theorem 2.1(i), we have

$$
\sum_{d(i, j, y) \geqslant 0}(d(i+1, j, y)-d(i, j, y)) P\left[S_{1} \geqslant d(i, j, y)\right]^{1 / v} \leqslant \sum_{n \in \mathbb{N}} P\left[S_{1} \geqslant n\right]^{1 / v}<\infty .
$$

Therefore, 


$$
\begin{aligned}
\sum_{d(i, j, y) \geqslant 0} \mathrm{E}\left[\left|L_{1}^{d(i+1, j, y)}-L_{1}^{d(i, j, y)}\right|^{p}\right] & \leqslant c(\beta) \sup _{d(i, j, y) \geqslant 0}(d(i+1, j, y)-d(i, j, y))^{(\beta-1) p / 2-1} \\
& \leqslant c(\beta) \epsilon_{k}^{(1-q / \beta)((\beta-1) p / 2-1)} .
\end{aligned}
$$

This implies that

$$
\mathrm{E} \sum_{(i, j)}\left|\Delta_{i j} L\right|^{p} \leqslant c(\beta) \epsilon_{k}^{\{(\beta-1) / 2\}\{q(p-2) / \beta)+p-2 /(\beta-1\}} .
$$

The exponent of $\epsilon_{k}$ is positive as soon as we choose $q$ strictly greater than $\beta\{2 /(\beta-1)-p\} /(p-2)$ and this is always possible since this last quantity is strictly smaller than $\beta$.

(ii) We consider a sequence of grids $\left(\Delta_{k}\right)_{k \in \mathbb{N}}$ defined as above, except that we will choose another value for $q$.

$$
\begin{aligned}
\mathrm{E} \sum_{(i, j)}\left|\Delta_{i j} L\right|^{p} & =\sum_{j}\left(t_{j+1}-t_{j}\right)^{p(\beta-1) / \beta} \int_{\mathbb{R}} P\left(X_{t_{j}} \in \mathrm{d} y\right) \sum_{i} \mathrm{E}\left[\left|L_{1}^{d(i+1, j, y)}-L_{1}^{d(i, j, y)}\right| p\right] \\
& \geqslant \epsilon_{k}^{q(p(\beta-1) / \beta-1)} \sum_{j}\left(t_{j+1}-t_{j}\right) \int_{a}^{b} P\left(X_{t_{j}} \in \mathrm{d} y\right) \sum_{i} \mathrm{E}\left[\left|L_{1}^{d(i+1, j, y)}-L_{1}^{d(i, j, y)}\right| p\right] .
\end{aligned}
$$

Now motivated by the proof of Theorem 2.1(ii), we write

$$
\int_{a}^{b} \int_{s}^{t} G_{k}(u, y) \mathrm{d} u \mathrm{~d} y=\epsilon_{k}^{q(p(\beta-1) / \beta-1)} \sum_{j}\left(t_{j+1}-t_{j}\right) \int_{a}^{b} P\left(X_{t_{j}} \in \mathrm{d} y\right) \mathrm{E}\left[\sum_{i}\left|L_{1}^{d(i+1, j, y)}-L_{1}^{d(i, j, y)}\right| p\right] \text {, }
$$

where

$$
G_{k}(u, y)=\epsilon_{k}^{q(p(\beta-1) / \beta-1)} \sum_{j} 1_{\left(t_{j}, t_{j+1}\right]}(u) p_{t_{j}}(y) \mathrm{E}\left[\sum_{i}\left|L_{1}^{d(i+1, j, y)}-L_{1}^{d(i, j, y)}\right| p\right] .
$$

Similarly, for a fixed pair $(u, y)$, there exists a unique sequence $(j(k))_{k}$ (actually $\left.(j(k, u))_{k}\right)$ such that $t_{j(k)}<u \leqslant t_{j(k)+1}$ for every $k$. Now if $y \in[a, b]$, then $(d(i, j(k), y))_{1 \leqslant i \leqslant n}$ is an ordered sequence such that $\sup _{i}(d(i+1, j(k), y)-d(i, j(k), y))$ tends to $0, d(1, j(k), y) \rightarrow-\infty$, and $d(n, j(k), y) \rightarrow+\infty$, as $k$ tends to $\infty$. Moreover, for any $i, u$ and $y$,

$$
3^{-\beta} \epsilon_{k}^{1-q / \beta} \leqslant d(i+1, j(k), y)-d(i, j(k), y) \leqslant 3 \epsilon_{k}^{1-q / \beta} .
$$

We want to find a lower bound for

$$
\epsilon_{k}^{q(p(\beta-1) / \beta-1)} \sum_{|d(i, j(k), y)| \leqslant M}\left|L_{1}^{d(i+1, j(k), y)}-L_{1}^{d(i, j(k), y)}\right|^{p} .
$$

In order to do so, we will use the following two results. The first was established by Barlow (1988) and Marcus and Rosen (1992a), this is the exact modulus of continuity of $L_{1}$, namely, 


$$
\lim _{\delta \rightarrow 0} \sup _{\substack{|x-y|<\delta \\|x|,|y| \leqslant M}} \frac{\left|L_{1}^{x}-L_{1}^{y}\right|}{|x-y|^{(\beta-1) / 2}\left(\log \left(\frac{1}{|x-y|}\right)\right)^{1 / 2}}=c(\beta)\left(\sup _{|z| \leqslant M} L_{1}^{z}\right)^{1 / 2} \text { a.s. }
$$

The second result is a refinement of (1) and was proved by Marcus and Rosen (1992b). Retaining the notation of the Introduction, and moreover assuming that the sequence of partitions $\left(\pi_{n}\right)_{n \in \mathbb{N}}$ is such that $\left|\pi_{n}\right|=o(1 / \log n)^{1 /(\beta-1)}$, then the convergence in (1) is almost sure.

To apply this result to the partitions $\left(d(i, j(k), y)_{i}\right.$ indexed by $k$, we take from now on

$$
\epsilon_{k}=\frac{1}{k}\left(\frac{1}{\log k}\right)^{1 /(\beta-1)(1-q / \beta)}
$$

We set

$$
Y(\delta)=\sup _{\substack{|x-y|<\delta \\|x|,|y| \leqslant M}} \frac{\left|L_{1}^{x}-L_{1}^{y}\right|}{|x-y|^{(\beta-1) / 2}\left(\log \left(\frac{1}{|x-y|}\right)\right)^{1 / 2}} .
$$

We now we remark that, for any $M>0$, we have

$$
\begin{aligned}
\sum_{i}\left|L_{1}^{d(i+1, j(k), y)}-L_{1}^{d(i, j(k), y)}\right| p & \geqslant \sum_{\mid d(i, j(k), y)<M}\left|L_{1}^{d(i+1, j(k), y)}-L_{1}^{d(i, j(k), y)}\right| p \\
& \geqslant \frac{\sum_{|d(i, j(k), y)|<M}\left|L_{1}^{d(i+1, j(k), y)}-L_{1}^{d(i, j(k), y)}\right| 2 /(\beta-1)}{\sup _{|d(i, j(k), y)|<M}\left|L_{1}^{d(i+1, j(k), y)}-L_{1}^{d(i, j(k), y)}\right| 2 /(\beta-1)-p},
\end{aligned}
$$

which leads to

$$
\begin{aligned}
& \epsilon_{k}^{q(p(\beta-1) / \beta-1)} \sum_{i}\left|L_{1}^{d(i+1, j(k), y)}-L_{1}^{d(i, j(k), y)}\right| p \geqslant c(\beta)\left[\sum_{|d(i, j(k), y)|<M}\left|L_{1}^{d(i+1, j(k), y)}-L_{1}^{d(i, j(k), y)}\right| 2 /(\beta-1)\right] \\
& \quad \times\left[Y\left(3 \epsilon_{k}^{1-q / \beta}\right)\right]^{p-2 /(\beta-1)} \epsilon_{k}^{\{(\beta-1) / 2\}\{q(p-2) / \beta+p-2 / \beta-1\}}\left|\log \left(\epsilon_{k}\right)\right|^{(p-2 /(\beta-1)) / 2} .
\end{aligned}
$$

By the two results just recalled, we know that, for any pair $(u, y)$,

$$
\begin{gathered}
\lim _{k \rightarrow \infty}\left[\sum_{|d(i, j(k), y)|<M}\left|L_{1}^{d(i+1, j(k), y)}-L_{1}^{d(i, j(k), y)}\right| 2 /(\beta-1)\right]\left[Y\left(3 \epsilon_{k}^{1-q / \beta}\right)\right]^{p-2 /(\beta-1)} \\
=c(\beta) \int_{-M}^{M}\left|L_{t}^{x}\right|^{1 /(\beta-1)} \mathrm{d} x\left(\sup _{|z| \leqslant M} L_{1}^{z}\right)^{p-2 /(\beta-1)} \text { a.s. }
\end{gathered}
$$

Since this last limit is almost surely strictly positive, the previous inequality shows us that 


$$
\lim _{k \rightarrow \infty} \epsilon_{k}^{q(p(\beta-1) / \beta-1)} \sum_{i}\left|L_{1}^{d(i+1, j(k), y)}-L_{1}^{d(i, j(k), y)}\right|^{p}=+\infty \text { a.s. }
$$

for any $q$ such that $0<q<\beta\{2 /(\beta-1)-p\} /(p-2)$.

Making use of the last argument in the proof of Theorem 2.1(ii), we obtain that, for almost every pair $(u, y)$ of $[s, t] \times[a, b], G_{k}(u, y)$ converges to $+\infty$ as $k$ tends to $\infty$. The same argument finally gives

$$
\int_{a}^{b} \int_{s}^{t} G_{k}(u, y) \mathrm{d} u \mathrm{~d} y \underset{k \rightarrow \infty}{\longrightarrow}+\infty
$$

\section{Quadratic variation of the local times for $\beta=2$}

Here $X$ is a Brownian motion. Our main tool is Tanaka's formula, which we formulate as

$$
\frac{1}{2} L_{t}^{x}=M(x, t)-V(x, t),
$$

where

$$
M(x, t)=\int_{0}^{t} 1_{\left(X_{s} \leqslant x\right)} \mathrm{d} X_{S}
$$

and

$$
V(x, t)=\left(X_{0}-x\right)^{-}-\left(X_{t}-x\right)^{-} .
$$

We start by computing the quadratic variation of $M$.

Theorem 3.1. Let $\left(\Delta_{k}\right)_{k \in \mathbb{N}}$ be a sequence of grids of $[a, b] \times[s, t]$ such that $\left|\Delta_{k}\right|$ converges to 0 as $k$ tends to $\infty$. Then we have

$$
\sum_{(i, j)}\left(\Delta_{i j} M\right)^{2} \underset{k \rightarrow \infty}{\stackrel{L^{2}}{\longrightarrow}} \int_{s}^{t} 1_{(a, b]}\left(X_{u}\right) \mathrm{d} u .
$$

Proof. We have to compute the limit as $k$ tends to $\infty$ of

$$
\sum_{(i, j)}\left(\int_{t_{j}}^{t_{j+1}} 1_{\left(x_{i}, x_{i+1}\right]}\left(X_{u}\right) \mathrm{d} X_{u}\right)^{2} .
$$

Instead of computing this limit directly (which is easy by Itô's calculus), it is more interesting to use a result of Bouleau (1982) which ensures, in particular, that for every continuous martingale $Z$ and every sequence of predictable partitions $\left(H_{i, k}, i \in \mathbb{N}\right)_{k \in \mathbb{N}}$ such that for $\left.\mathrm{d}[Z]_{u} \times \mathrm{d} P\right)$-almost every $\left(\omega_{0}, t_{0}\right)$,

$$
\begin{aligned}
& \limsup _{k \rightarrow \infty} H_{i, k}\left(\omega_{0}, \cdot\right)=0 \mathrm{~d}[Z]_{u} \text {-a.s. } \\
& \begin{array}{c}
k \rightarrow \infty \\
\left(\omega_{0}, t_{0}\right) \in H_{i, k}
\end{array}
\end{aligned}
$$


we then have

$$
\sum_{i}\left(\int_{0}^{\infty} H_{i, k}(s) \mathrm{d} Z_{s}\right)^{2} \underset{k \rightarrow \infty}{\stackrel{L^{2}}{\longrightarrow}}[Z]_{\infty} .
$$

Choosing

$$
Z_{u}=\int_{0}^{u} 1_{[s, t]}(v) 1_{[a, b]}\left(X_{v}\right) \mathrm{d} X_{v}
$$

and

$$
H_{i j, k}(\omega, v)=1_{\left(t_{j}, t_{j+1}\right]}(v) 1_{\left(x_{i}, x_{i+1}\right]}\left(B_{v}(\omega)\right),
$$

we obtain Theorem 3.1.

Remark 3.1. For a fixed $b \in \mathbb{R}$, we know that $(M(b, t), t \geqslant 0)$ is a continuous local martingale with quadratic variation on any interval $[s, t]$ equal to

$$
[M(b, \cdot)]_{t}-[M(b, \cdot)]_{s}=\int_{s}^{t} 1_{\left(X_{u} \leqslant b\right)} \mathrm{d} u .
$$

For a fixed $t \in \mathbb{R}^{*}$, Perkins (1982) has shown that $\left(L_{t}^{x}, x \in \mathbb{R}\right)$ is a continuous semimartingale with respect to the excursion field. Since the process $(V(x, t), x \in \mathbb{R})$ is a process of bounded variation, adapted to the excursion field, $(M(x, t), x \in \mathbb{R})$ is also a continuous semimartingale with the same quadratic variation as $L_{t}$ on any interval $[a, b]$ (computed also by Bouleau and Yor 1981). We obtain

$$
[M(\cdot, t)]_{b}-[M(\cdot, t)]_{a}=\int_{0}^{t} 1_{\left(a<X_{u} \leqslant b\right)} \mathrm{d} u .
$$

The motivation of Bouleau (1982) was actually to give an explanation to the following remarkable equality:

$$
\begin{aligned}
& {[M(b, \cdot)]_{t}-[M(b, \cdot)]_{s}-[M(a, \cdot)]_{t}+[M(a, \cdot)]_{s}} \\
& \quad=[M(\cdot, t)]_{b}-[M(\cdot, t)]_{a}-[M(\cdot, s)]_{b}+[M(\cdot, s)]_{a} .
\end{aligned}
$$

We have used his explanation to prove that each member of this equality is also equal to the quadratic variation of $M$ on $[a, b] \times[s, t]$.

Moreover, one can easily prove that $M$ and $M^{2}$ are also doubly indexed semimartingales, and that $M$ and $\left(M^{2}(x, t)-\int_{0}^{t} 1_{\left(X_{u} \leqslant x\right)} \mathrm{d} u ; x \in \mathbb{R}, t \geqslant 0\right)$ are weak martingales - see Meyer (1981) for the precise definitions. All these properties make of $M$ a very nice example of a doubly indexed semimartingale. However, in Eisenbaum (1998) it is shown that as a doubly indexed process $L$ does not share the semimartingale properties of $M$.

Remark 3.2. If $V$ were a doubly indexed process of bounded variation, then we would obtain immediately that the quadratic variation of $L$ is four times the quadratic variation of $M$. But $V$ is not of bounded variation. Indeed, thanks to Tanaka's formula, we know that 
$\left.\left(\left(X_{t}-b\right)^{-}-\left(X_{t}-a\right)^{-}\right), t \geqslant 0\right)$ is a continuous semimartingale, hence it has finite quadratic variation on $[0,1]$ and thus infinite variation on $[0,1]$. We then note that

$$
\sum_{(i, j)}\left|\Delta_{i j} V\right| \geqslant \sum_{j}\left|\left(X_{t_{j+1}}-b\right)^{-}-\left(X_{t_{j}}-b\right)^{-}-\left(X_{t_{j+1}}-a\right)^{-}+\left(X_{t_{j}}-a\right)^{-}\right| .
$$

Nevertheless, the next theorem will show that, in a way, $V$ has a quadratic variation equal to 0 on any rectangle, and this will be sufficient to establish that the quadratic variation of $L$ is four times the quadratic variation of $M$.

Theorem 3.2. Let $\left(\Delta_{k}\right)_{k \in \mathbb{N}}$ be a sequence of grids of $[a, b] \times[s, t]$, where, for each $k$, $\Delta_{k}=\left\{\left(x_{i}, s_{j}\right), 1 \leqslant i \leqslant n, 1 \leqslant j \leqslant m\right\}$. We suppose that $\left|\Delta_{k}\right|$ and

$$
\sup _{\left(x_{i}, s_{j}\right) \in \Delta_{k}}\left|x_{i+1}-x_{i}\right| / \sqrt{t_{j+1}-t_{j}}
$$

both converge to 0 as $k$ tends to $\infty$. We then have

(i) $\sum_{(i, j)}\left(\Delta_{i j} V\right)^{2} \underset{k \rightarrow \infty}{\stackrel{L^{1}}{\longrightarrow}} 0$

(ii) $\sum_{(i, j)}\left(\Delta_{i j} L\right)^{2} \underset{k \rightarrow \infty}{\stackrel{L^{1}}{\longrightarrow}} 4 \int_{s}^{t} 1_{(a, b]}\left(X_{u}\right) \mathrm{d} u$.

In order to prove Theorem 3.2, we establish the following lemma:

Lemma 3.3. Under the hypotheses of Theorem 3.1,

$$
\mathrm{E}\left[\sum_{(i, j)}\left(\Delta_{i j} L\right)^{2}\right] \underset{k \rightarrow \infty}{\longrightarrow} 4 \mathrm{E}\left[\int_{s}^{t} 1_{(a, b]}\left(X_{v}\right) \mathrm{d} v\right] .
$$

Proof of Lemma 3.3. By successively applying the Markov property of $X$ at time $t_{j}$ and its scaling property, we obtain

$$
\begin{aligned}
\mathrm{E}\left[\sum_{(i, j)}\left(\Delta_{i j} L\right)^{2}\right] & =\mathrm{E}\left[\sum_{(i, j)}\left(L_{t_{j+1}-t_{j}}^{x_{i+1}}-L_{t_{j+1}-t_{j}}^{x_{i}}\right)^{2} \circ \theta_{t_{j}}\right] \\
& =\sum_{j} \mathrm{E}\left[\mathrm{E}\left[\left(\sum_{i}\left(L_{t_{j+1}-t_{j}}^{x_{i+1}}-L_{t_{j+1}-t_{j}}^{x_{i}}\right)^{2}\right) \circ \theta_{t_{j}} \mid \mathscr{F}_{t_{j}}\right]\right] \\
& =\sum_{j} \int_{\mathbb{R}} P\left(X_{t_{j}} \in \mathrm{d} y\right) \mathrm{E}\left[\sum_{i}\left(L_{t_{j+1}-t_{j}}^{x_{i+1}-y}-L_{t_{j+1}-t_{j}}^{x_{i}-y}\right)^{2}\right] \\
& =\sum_{j}\left(t_{j+1}-t_{j}\right) \int_{\mathbb{R}} P\left(X_{t_{j}} \in \mathrm{d} y\right) \sum_{i} \mathrm{E}\left[\left(L_{1}^{d(i+1, j, y)}-L_{1}^{d(i, j, y)}\right)^{2}\right] .
\end{aligned}
$$


We thus obtain

$$
\mathrm{E}\left[\sum_{(i, j)}\left(\Delta_{i j} L\right)^{2}\right]=\int_{\mathbb{R}} \mathrm{d} y \int_{s}^{t} \mathrm{~d} u F_{k}(u, y)
$$

with

$$
F_{k}(u, y)=\sum_{j} 1_{\left(t_{j}, t_{j+1}\right]}(u) \frac{1}{\sqrt{2 \pi t_{j}}} \mathrm{e}^{-y^{2} / 2 t_{j}} \mathrm{E}\left[\sum_{i}\left(L_{1}^{d(i+1, j, y)}-L_{1}^{d(i, j, y)}\right) 2\right] .
$$

For a fixed pair $(u, y)$, there exists a unique sequence $(j(k))_{k}$ (actually $\left.(j(k, u))_{k}\right)$ such that $t_{j(k)}<u \leqslant t_{j(k)+1}$ for every $k$. Either $y \in[a, b]$, in which case $(d(i, j(k), y))_{1 \leqslant i \leqslant n}$ is a partition such that $\sup _{i}(d(i+1, j(k), y)-d(i, j(k), y))$ tends to $0, d(1, j(k), n) \rightarrow-\infty$, and $d(n, j(k), y) \rightarrow+\infty$, as $k$ tends to $\infty$, and consequently

$$
\sum_{i}\left(L_{1}^{d(i+1, j(k), y)}-L_{1}^{d(i, j(k), y)}\right)^{2} \stackrel{L^{2}}{\longrightarrow} 4 \int_{0}^{1} 1_{(-\infty,+\infty)}\left(X_{v}\right) \mathrm{d} v,
$$

which implies that

$$
\mathrm{E}\left[\sum_{i}\left(L_{1}^{d(i+1, j(k), y)}-L_{1}^{d(i, j(k), y)}\right)^{2}\right] \underset{k \rightarrow \infty}{\longrightarrow} 4
$$

or $y<a(y>b)$, in which case the partition $(d(i, j(k), y))_{1 \leqslant i \leqslant n}$ converges to $-\infty(+\infty)$ and thus

$$
\mathrm{E}\left[\sum_{i}\left(L_{1}^{d(i+1, j(k), y)}-L_{1}^{d(i, j(k), y)}\right)^{2}\right] \underset{k \rightarrow \infty}{\longrightarrow} 0 .
$$

In summary, we obtain

$$
F_{k}(u, y) \underset{k \rightarrow \infty}{\longrightarrow} 1_{[a, b]}(y) \frac{4}{\sqrt{2 \pi u}} \mathrm{e}^{-y^{2} / 2 u} .
$$

To apply the Lebesgue dominated convergence theorem, we remark that the set

$$
\left\{\sum_{i}\left(L_{1}^{y_{i+1}}-L_{1}^{y_{i}}\right)^{2},\left(y_{i}\right)_{i} \text { a partition of }(-\infty,+\infty)\right\}
$$

is bounded in $L^{1}$ (see, for example, Proposition 3 of Bouleau 1982). Consequently, we obtain

$$
\int_{\mathbb{R}} \mathrm{d} y \int_{s}^{t} \mathrm{~d} u F_{k}(u, y) \underset{k \rightarrow \infty}{\longrightarrow} \int_{\mathbb{R}} \mathrm{d} y \int_{s}^{t} \mathrm{~d} u 1_{[a, b]}(y) \frac{4}{\sqrt{2 \pi u}} \mathrm{e}^{-y^{2} / 2 u}
$$

and this last limit is equal to $4 \int_{s}^{t} \mathrm{~d} u P\left(a \leqslant X_{u} \leqslant b\right)$.

We now use the following result, established in Eisenbaum (1998), which is a simple consequence of Tanaka's formula, namely, 


$$
L_{t}^{x}=M(x, t)+N(x, t), \quad x \in \mathbb{R}, 0 \leqslant t \leqslant 1,
$$

where $N(x, t)=\int_{1-t}^{1} 1_{\left(X_{1-s} \leqslant x\right)} \mathrm{d} X_{1-s}$. Hence, we have

$$
\sum_{i, j}\left(\Delta_{i j} L\right)^{2}=\sum_{i, j}\left(\Delta_{i j} M\right)^{2}+\sum_{i, j}\left(\Delta_{i j} N\right)^{2}+2 \sum_{i, j}\left(\Delta_{i j} N\right)\left(\Delta_{i j} M\right) .
$$

Using the same argument as in Eisenbaum (1998), we see that we also have

$$
2 V(x, t)=M(x, t)-N(x, t),
$$

and similarly

$$
4 \sum_{i, j}\left(\Delta_{i j} V\right)^{2}=\sum_{i, j}\left(\Delta_{i j} M\right)^{2}+\sum_{i, j}\left(\Delta_{i j} N\right)^{2}-2 \sum_{i, j}\left(\Delta_{i j} N\right)\left(\Delta_{i j} M\right) .
$$

We recall that the one-dimensional semimartingale $\left(X_{1-s}, 0 \leqslant s \leqslant 1\right)$ admits the decomposition

$$
X_{1-s}=X_{1}+W_{t}-\int_{0}^{t} \frac{X_{1-s}}{1-s} \mathrm{~d} s
$$

where $W$ is a Brownian motion starting from 0 . To compute the quadratic variation of $N$, we first note that the process

$$
\left(\int_{0}^{t} 1_{\left(X_{1-s} \leqslant x\right)} \frac{X_{1-s}}{1-s} \mathrm{~d} s, x \in \mathbb{R}, 0 \leqslant t \leqslant 1\right)
$$

has bounded variation on any finite rectangle. Consequently, using the same argument as for $M$, we know that

$$
\sum_{i, j}\left(\Delta_{i j} N\right)^{2} \underset{k \rightarrow \infty}{\stackrel{L^{1}}{\longrightarrow}} \int_{s}^{t} 1_{[a, b]}\left(X_{u}\right) \mathrm{d} u .
$$

Therefore, Lemma 3.3 and (3) together give

$$
\mathrm{E}\left[\sum_{i, j}\left(\Delta_{i j} N\right)\left(\Delta_{i j} M\right)\right] \underset{k \rightarrow \infty}{\longrightarrow} \int_{S}^{t} 1_{[a, b]}\left(X_{u}\right) \mathrm{d} u .
$$

Thanks to (4), we obtain

$$
\mathrm{E}\left[\sum_{i, j}\left(\Delta_{i j} V\right)^{2}\right] \underset{k \rightarrow \infty}{\longrightarrow} 0
$$

which is equivalent to

$$
\sum_{i, j}\left(\Delta_{i j} V\right)^{2} \underset{k \rightarrow \infty}{\stackrel{L^{1}}{\longrightarrow}} 0
$$

Thus (i) is proved.

Going back to (4), we hence know that 


$$
\sum_{i, j}\left(\Delta_{i j} N\right)\left(\Delta_{i j} M\right) \underset{k \rightarrow \infty}{\stackrel{L^{1}}{\longrightarrow}} \int_{s}^{t} 1_{[a, b]}\left(X_{u}\right) \mathrm{d} u
$$

Assertion (ii) is then proved thanks to (3).

\section{Rewriting of an Itô formula}

Let $F$ be an absolutely continuous function on $\mathbb{R}$ with a locally bounded measurable derivative $F^{\prime}$. For any pair of reals $(a, b)$, we have

$$
F(b)=F(a)+\int_{\mathbb{R}} F^{\prime}(x) \mathrm{d}_{x}\left((a-x)^{-}-(b-x)^{-}\right) .
$$

In particular, for the Brownian motion $X$, we have

$$
F\left(X_{t}\right)=F\left(X_{0}\right)+\int_{\mathbb{R}} F^{\prime}(x) \mathrm{d}_{x} V(x, t) .
$$

We now consider a function $F$ defined on $\mathbb{R} \times \mathbb{R}_{+}$and seek an analogous representation for $F\left(X_{t}, t\right)-F\left(X_{0}, 0\right)$. To this end, we need the following results established in Eisenbaum (1998).

For $f$ a measurable function from $\mathbb{R} \times[0,1]$ into $\mathbb{R}$, we define the norm $\|\cdot\|$ by

$$
\|f\|=2\left(\int_{0}^{1} \int_{\mathbb{R}} f^{2}(x, s) \mathrm{e}^{-x^{2} / 2 s} \frac{\mathrm{d} x \mathrm{~d} s}{\sqrt{2 \pi s}}\right)^{1 / 2}+\int_{0}^{1} \int_{\mathbb{R}}|x f(x, s)| \mathrm{e}^{-x^{2} / 2 s} \frac{\mathrm{d} x \mathrm{~d} s}{s \sqrt{2 \pi s}} .
$$

Let $\mathscr{H}$ be the set of functions $f$ such that $\|f\|<\infty$.

In Eisenbaum (1998), it is shown that integration with respect to $L$ is possible in the following sense: let $f_{\Delta}$ be an elementary function on $\mathbb{R} \times \mathbb{R}^{+}$, meaning that

$$
f_{\Delta}(x, t)=\sum_{\left(x_{i}, s_{j}\right) \in \Delta} f_{i, j} 1_{\left(x_{i}, x_{i+1}\right]}(x) 1_{\left(s_{j}, s_{j+1}\right]}(t),
$$

where $\Delta=\left\{\left(x_{i}, s_{j}\right), 1 \leqslant i \leqslant n, 1 \leqslant j \leqslant m\right\}$ is an $\mathbb{R} \times[0,1]$ grid, and, for every $(i, j), f_{i j}$ is in $\mathbb{R}$. For such a function, integration with respect to $L$ is defined by

$$
\int_{0}^{+\infty} \int_{\mathbb{R}} f_{\Delta}(x, s) \mathrm{d} L_{s}^{x}=\sum_{\left(x_{i}, s_{j}\right) \in \Delta} f_{i, j}\left(\Delta_{i j} L\right) .
$$

Let $f$ be an element of $\mathscr{H}$. For any sequence of elementary functions $\left(f_{\Delta_{k}}\right)_{k \in \mathbb{N}}$ converging to $f$ in $\mathscr{H}$, the sequence $\left(\int_{0}^{1} \int_{\mathbb{R}} f_{\Delta_{k}}(x, s) \mathrm{d} L_{s}^{x}\right)_{k \in \mathbb{N}}$ converges in $L^{1}$. The limit obtained does not depend of the choice of the sequence $\left(f_{\Delta_{k}}\right)$ and represents the integral $\int_{0}^{1} \int_{\mathbb{R}} f(x, s) \mathrm{d} L_{s}^{x}$.

We can also define $\int_{0}^{1} \int_{\mathbb{R}} f(x, s) \mathrm{d} M(x, s)$ for a deterministic function $f$ in a similar sense to that above. More precisely, we have 


$$
\int_{0}^{1} \int_{\mathbb{R}} f_{\Delta}(x, s) \mathrm{d} M(x, s)=\sum_{\left(x_{i}, s_{j}\right) \in \Delta} f_{i, j}\left(\Delta_{i j} M\right)=\int_{0}^{1} f_{\Delta}\left(X_{s}, s\right) \mathrm{d} X_{s} .
$$

Let $f$ be a function such that

$$
\int_{0}^{1} \int_{\mathbb{R}} f^{2}(x, s) \mathrm{e}^{-x^{2} / 2 s} \frac{\mathrm{d} x \mathrm{~d} s}{\sqrt{2 \pi s}}<\infty .
$$

Then, for every sequence of elementary deterministic functions $\left(f_{\Delta_{k}}\right)_{k \in \mathbb{N}}$ converging simply to $f$, we know that

$$
\int_{0}^{1} f_{\Delta_{k}}\left(X_{s}, s\right) \mathrm{d} X_{s} \underset{k \rightarrow \infty}{\stackrel{L^{2}}{\longrightarrow}} \int_{0}^{1} f\left(X_{s}, s\right) \mathrm{d} X_{s}
$$

Consequently, we obtain

$$
\int_{0}^{1} \int_{\mathbb{R}} f(x, s) \mathrm{d} M(x, s)=\int_{0}^{1} f\left(X_{s}, s\right) \mathrm{d} X_{s} .
$$

We now introduce the process $(A(x, t) ; x \in \mathbb{R}, t \geqslant 0)$ defined by $A(x, t)=\int_{0}^{t} 1_{\left(X_{s} \leqslant x\right)} \mathrm{d} s$. We note that $A$ has bounded variation on any bounded rectangle and similarly satisfies

$$
\int_{0}^{t} \int_{\mathbb{R}} f(x, s) \mathrm{d} A(x, s)=\int_{0}^{t} f\left(X_{s}, s\right) \mathrm{d} s
$$

for any function $f$ such that

$$
\int_{0}^{1} \int_{\mathbb{R}}|f(x, s)| \mathrm{e}^{-x^{2} / 2 s} \frac{\mathrm{d} x \mathrm{~d} s}{\sqrt{2 \pi s}}<\infty .
$$

We can then obtain the following representation for $F\left(X_{t}, t\right)-F\left(X_{0}, 0\right)$ :

Theorem 4.1. For $F$ defined on $\mathbb{R} \times[0,1]$ such that $\partial F / \partial x$ and $\partial F / \partial t$ exist as RadonNikodym derivatives, $\partial F / \partial x$ is in $\mathscr{H}$ and

$$
\int_{0}^{1} \int_{\mathbb{R}} \frac{\partial F}{\partial t}(x, s) \mid \frac{\mathrm{d} x \mathrm{~d} s}{s \sqrt{2 \pi s}}<\infty
$$

we have

$$
F\left(X_{t}, t\right)=F\left(X_{0}, 0\right)+\int_{0}^{t} \int_{\mathbb{R}} \frac{\partial F}{\partial s}(x, s) \mathrm{d} A(x, s)+\int_{0}^{t} \int_{\mathbb{R}} \frac{\partial F}{\partial x}(x, s) \mathrm{d} V(x, s) .
$$

Proof. Since we have $V=M-\frac{1}{2} L$, integration with respect to $V$ is also possible for $f$ in $\mathscr{H}$ and satisfies

$$
\int_{0}^{t} \int_{\mathbb{R}} f(x, s) \mathrm{d} V(x, s)=\int_{0}^{t} f\left(X_{s}, s\right) \mathrm{d} X_{s}-\frac{1}{2} \int_{0}^{t} \int_{\mathbb{R}} f(x, s) \mathrm{d} L_{s}^{x} .
$$

We recall now an Itô formula established in Eisenbaum (1998). Under the hypotheses of Theorem 4.1, we have 


$$
F\left(X_{t}, t\right)=F\left(X_{0}, 0\right)+\int_{0}^{t} \frac{\partial F}{\partial s}\left(X_{s}, s\right) \mathrm{d} s+\int_{0}^{t} \frac{\partial F}{\partial x}\left(X_{s}, s\right) \mathrm{d} X_{s}-\frac{1}{2} \int_{0}^{t} \int_{\mathbb{R}} \frac{\partial F}{\partial x}(x, s) \mathrm{d} L_{s}^{x} .
$$

Now using (6) and (7), Theorem 4.1 is proved.

\section{References}

Barlow, M.T. (1988) Necessary and sufficient conditions for the continuity of local time. Ann. Probab., 16, 1389-1427.

Bertoin, J. (1996) Lévy Processes. Cambridge: Cambridge University Press.

Bouleau, N. (1982) Sur la variation quadratique de certaines mesures vectorielles. Z. Wahrscheinlichkeitstheorie Verw. Geb., 61, 261-270.

Bouleau, N. and Yor, M. (1981) Sur la variation quadratique de temps locaux de certaines semimartingales. C. R. Acad. Sci. Paris Sér. I, 292, 491-492.

Boylan, E.S. (1964) Local times for a class of Markov processes. Illinois J. Math., 8, 19-39.

Eisenbaum, N. (1998) Integration with respect to local time. Potential Anal. To appear.

Marcus, M.B. and Rosen, J. (1992a) Sample path properties of the local times of strongly symmetric Markov processes via Gaussian processes. Ann. Probab., 20, 1603-1684.

Marcus, M.B. and Rosen, J. (1992b) p-variation of the local times of symmetric stable processes and of Gaussian processes with stationary increments. Ann. Probab., 20, 1685-1713.

Meyer, P.A. (1981) Théorie élémentaire des processus à deux indices. In H. Korezlioglu, G. Mazziotto and J. Szpirglas (eds), Processus Aléatoires à Deux Indices, Lecture Notes in Math. 863, pp. 139. Berlin: Springer-Verlag.

Perkins, E. (1982) Local time is a semi-martingale. Z. Wahrscheinlichkeitstheorie Verw. Geb., 60, 79117.

Rogers, L.C.G. and Walsh, J.B. (1991) Local time and stochastic area integrals. Ann. Probab., 19, $457-482$.

Walsh, J.B. (1983) Stochastic integration with respect to local time. In E. Çinlar, K.L. Chung and R.K. Getoor (eds), Stochastic Processes, Progr. Probab. Statist., 5, pp. 237-302. Boston: Birkhäuser.

Received December 1998 and revised November 1999 